$5-1-2010$

\title{
DNA Polymerases: Perfect Enzymes for An Imperfect World
}

Anthony J. Berdis

Cleveland State University, A.BERDIS@csuohio.edu

Follow this and additional works at: https://engagedscholarship.csuohio.edu/scichem_facpub

Part of the Biochemistry Commons, and the Chemistry Commons

How does access to this work benefit you? Let us know!

\section{Recommended Citation}

Berdis, Anthony J., "DNA Polymerases: Perfect Enzymes for An Imperfect World" (2010). Chemistry Faculty Publications. 185.

https://engagedscholarship.csuohio.edu/scichem_facpub/185

This Article is brought to you for free and open access by the Chemistry Department at EngagedScholarship@CSU. It has been accepted for inclusion in Chemistry Faculty Publications by an authorized administrator of EngagedScholarship@CSU. For more information, please contact library.es@csuohio.edu. 


\section{DNA polymerases: Perfect enzymes for an imperfect world}

DNA is the molecule of life. The intricate and unique spatial arrangement of four nucleobases (adenine, cytosine, guanine, and thymine) provides the genetic blueprint for all forms of life on this planet. Indeed, great strides have been made to decipher the complete genomes from organisms ranging from simple viruses and bacteria to more complex eukaryotes including mammals. Of particular relevance is the blueprint of the human genome as it contains information defining "what" we are as a species as well as "who" we are as individuals. By annotating the exact chemical sequence of the 3 billion base pairs in our genome, completion of the Human Genome Project in 2003 was a monumental achievement since it identified the $\sim 25,000$ genes that define our biological nature. The hope of molecular medicine is that this chemical information can be translated into technologies that can provide accurate personal histories encompass ing our past, present, and even future life experiences. Although we have just begun to decipher this massive amount of genetic information, the fruits of this endeavor are starting to be realized. For example, the Cancer Genome Atlas (cancergenome.nih.gov) has begun to identify nuances in the DNA sequences of different types of cancers as well as differences between sub types of the same malignancy. Eventually, deciphering an individual's nuclear and mitochondrial genome will generate diagnostic markers that can identify and predict the onset of various diseases. These efforts will also generate prognostic indicators for therapeutic intervention and clinical outcomes. While these predictions may seem like science fiction, there are existing nucleic acid based technologies that have already had an enormous impact on basic science and translation research efforts. For instance, the ability to selectively and efficiently introduce another organism's genomic DNA into a bacterial cell was widely considered to be an improbable feat just forty years ago. Today, introducing DNA into prokaryotic and eukaryotic cells is performed on a routine basis. In fact, this technology has revolutionized many scientific fields including microbiology, biochemistry, genetics, struc tural biology, and pharmacology to name just a few.

While it is clear that DNA technologies have enhanced our lives in numerous ways, we tend to take for granted the enzymes that are responsible for efficiently and accurately copying this precious material. These enzymes, denoted as DNA polymerases, catalyze the incorporation of mononucleotides into a growing primer using a DNA template as a guide for directing each incorporation event. DNA polymerases are remarkable enzymes as most perform the repetitive cycle of nucleotide binding, base pairing, phosphodiester bond formation, product release, and movement to the next templating position with incredibly fast rates as well as with impeccable precision and accuracy. Polymerases play essential cellular roles by copying genomic material so that it can be passed from a mother cell to its progeny. In addition, they play a pre eminent role in preserving its integrity. Without these wonderful biological machines, life as we know it would not exist.

This Special Thematic Issue explores the molecular nature of DNA polymerases as extraordinary biological catalysts. These articles discuss numerous contemporary issues of DNA polymeri zation, paying particular attention to the dynamic cellular roles and biochemical function of DNA polymerases, and how their structure and mechanism impact their cellular roles. Although not formally demarcated, the contents of this Special Issue are loosely arranged into three (3) distinct sub categories that correspond to mecha nistic studies of faithful DNA polymerization, studies of "special ized" DNA polymerases that function on damaged DNA, and DNA polymerases that are of therapeutic importance against various diseases.

The first six articles of this edition focus on understanding how high fidelity DNA polymerases replicate genomic DNA with incredible efficiency and accuracy. Emphasis is placed on the use of specialized techniques to study polymerase activity, the mechanistic information derived from applying these techniques, and correlating this mechanistic knowledge with available structural information of various DNA polymerases complexed with DNA in the absence and presence of nucleotide substrates. The first article by Catherine Joyce (Yale University) provides a comprehensive overview of techniques and methodologies currently employed to accurately measure polymerase activity. Her article places special emphasis on rapid 
chemical quench and fluorescence based assays that can identify the roles of key kinetic steps along the DNA polymerization pathway. The article by Kenneth Johnson (University of Texas at Austin) expands on this and describes how many of these techniques are used to define our current understanding of the kinetic and thermodynamic basis for nucleotide selection. In addition, Ken discusses practical methodolo gies to define the fidelity of nucleotide incorporation and then provides examples of how this information yielded key mechanistic interpretations of DNA polymerase function, especially within the context of existing structural data. Linda Reha Krantz (University of Alberta) provides an excellent overview of how high fidelity DNA polymerases use their intrinsic exonuclease proofreading activity to maintain genomic fidelity. In addition to defining the intricacies of this kinetic pathway, she describes various cellular roles of exonu clease proofreading, particularly with respect to coordinating this activity with DNA repair and apoptotic pathways to ensure overall genomic fidelity. Irene Lee and I (Case Western Reserve University) describe how non natural nucleotides, i.e., nucleotides that lack functional groups associated with classical hydrogen bonding inter actions, can be used to probe the mechanism of correct and incorrect DNA synthesis. This article provides a sweeping overview of non natural analogs that have been used to explore polymerase function. Collectively, the results generated using these analogs have lead to the development of several unique models (steric guidance, shape complementary, desolvation, positive/negative selection, and base stacking interactions) that can account for polymerization efficiency and fidelity. While DNA polymerases are considered to be the "workhorses" for DNA synthesis, efficient and faithful replication within the cell depends upon a coalition of distinct protein activities that function in a collaborative and highly coordinated fashion. The next two articles examine how high fidelity polymerases are regulated by dynamic interactions with other cellular proteins denoted as accessory proteins. The article by Zhuang and Ai (University of Delaware) describes the essential role of "sliding clamp" proteins that serve as processivity factors and coordinate highly efficient DNA synthesis by high fidelity DNA polymerases. In addition, these authors lay the foundation for understanding how "sliding clamp" proteins interact with other low fidelity DNA polymerases to maintain the continuity of DNA synthesis when damaged DNA is encountered. Finally, Stephen Benkovic et al. (The Pennsylvania State University) describe research using single mole cule techniques to study the molecular interactions of the ensemble of proteins associated with the replication fork. They provide compelling evidence that single molecule techniques can efficiently and accu rately define transient interactions amongst various protein compo nents of the moving replication fork. Citing results obtained using this technique, the authors compare and contrast the dynamics of leading and lagging strand DNA synthesis from various model replication systems including bacteriophage T4 and E. coli.

Even under ideal cellular conditions, the ability of a polymerase to replicate millions of base pairs without introducing errors is a daunting task. Indeed, DNA is a highly reactive molecule that is susceptible to various modifications that can occur when cells are exposed to stressful conditions, i.e., oxidative stress, DNA damaging agents, etc. Modifications to DNA place additional strains on the activity of DNA polymerases, especially with their ability to maintain continuous and faithful DNA synthesis. Fortunately, cells have developed sophisticated ways to cope with various DNA lesions. One fascinating cellular strategy is to replicate beyond the lesion by employing the service of various "specialized" DNA polymerases. The focus of the next five manuscripts is to understand the role and molecular mechanisms by which several of these "specialized" DNA polymerases function to replicate damaged DNA. From a historical perspective, these DNA polymerases were originally classified as "error prone" since they have a propensity to misincorporate a wrong nucleotide opposite undamaged DNA with alarmingly high frequen cies. It has since been uncovered that these polymerases are instrumental in maintaining genomic fidelity since they can correctly by pass various DNA lesions with remarkably high accuracy. M. Todd Washington (University of Iowa) and colleagues will first discuss how these "specialized" polymerases differ from their high fidelity counterparts that replicate unmodified DNA. Several models are described that range from the ability to tolerate distorted templates and primers to the use of Hoogsteen base pairing interactions to the use of active site amino acids that guide the incorporation of selective nucleotides. The article by Janice Pata (Wadsworth Center) provides deeper insight into these models by describing the structural features of several members of "specialized" DNA polymerases. Of particular relevance are structural distinctions in the molecular architecture of Y family polymerases with other DNA polymerases that display higher replicative fidelity. Jennifer Yamtich and Joann Sweasy (Yale University) provide a comprehensive overview of several members of the $X$ family of eukaryotic DNA polymerases, including polymerase beta (pol $\beta$ ), polymerase lambda (pol $\lambda$ ), and polymerase mu (pol $\mu$ ). Although these polymerases are structurally similar to each other, they display different biochemical properties, especially with DNA interactions that influence their cellular functions during DNA repair and lesion by pass. Ed Motea and I (Case Western Reserve University) discuss a very unique DNA polymerase, designated terminal deox ynucleotidyl transferase (TdT), that performs DNA synthesis using only single stranded DNA as the substrate. This chapter reviews the biological rationale for synthesizing DNA without guidance from a templating strand, and then examines the biophysical and biochem ical properties present in TdT that allow it to perform template independent DNA synthesis. Collectively, these four articles make it clear that cells possess multiple DNA polymerases that display different activities and fidelities. The obvious question is how their activities are controlled at the cellular level. Mark Sutton (University of Buffalo) provides some answers to this conundrum by summarizing models that account for the regulation of diverse DNA polymerases. These models, based on biochemical and genetic evidence, provide a rationale for how a proper balance between faithful replication versus pro mutagenic DNA synthesis is achieved at the organismal level. A strong emphasis is placed on describing models for how DNA polymerases switch places with each other at the replication fork.

DNA polymerases are essential for the survival and proliferation of all organisms. As a consequence, inhibiting their activity represents a viable strategy for therapeutic intervention, especially against hyperproliferative diseases such as cancer, autoimmune disorders, and viral/microbial infections. The final five papers describe DNA polymerases that are of therapeutic importance against these diseases. Gundren Stengel and Robert Kuchta (University of Colorado) will first discuss the mechanism of DNA primase, a unique polymerase that synthesizes short RNA primers that are necessary to commence lagging strand DNA synthesis. While the need to initiate de novo DNA synthesis is universal, these authors provide compelling evidence that there are significant mechanistic and structural differences amongst DNA primases from various organisms. These differences provide a rationale for therapeutic interventions, particularly against patho genic organisms such as herpes simplex virus. After describing how DNA polymerases are used to initiate DNA synthesis, Michael Jarstfers (University of North Carolina) and colleagues will discuss problems associated with "terminating" DNA synthesis. Their article will describe the role of telomerase in replicating the ends of linear, chromosomal DNA. Since telomerase activity is a common feature in cancer development, understanding the mechanism of this enzyme is essential to develop selective therapeutic agents. Toward this goal, these authors provide new insights into the roles of telomerase in human biology by discuss the structure and mechanism of this polymerase. Human immunodeficiency virus type 1 reverse tran scriptase (HIV $1 \mathrm{RT}$ ) is another well established therapeutic target. This polymerase catalyzes synthesis of double stranded DNA from 
the single stranded viral RNA genome. Le Grice (National Cancer Institute) and colleagues will discuss the mechanism of this fascinating enzyme which uses both polymerizing and hydrolytic functions to replicate RNA and DNA. These authors describe recent results obtained using single molecule spectroscopy techniques to monitor the reverse transcription process with higher resolution than conventional ensemble experiments. This information provides a new area in drug discovery that focuses on interfering with polymerase motion on nucleic acid rather than targeting the active site of the polymerase using nucleotide analogs. Christopher Bailey and Karen Anderson (Yale University) discuss polymerase gamma ( pol $\gamma$ ), the eukaryotic DNA polymerase that is responsible for replicating mitochondrial DNA. In addition to describing the kinetic character ization of this enzyme, these authors highlight key mechanistic and structural information as it relates to clinically relevant issues involving toxicity caused by nucleoside analogs used to treat HIV as well as mitochondrial disorders such as progressive external opthalmoplegia. Finally, McKenna (University of Southern California) and colleagues provide an interesting article describing novel nucleoside analogs that have the potential to act as anti viral and anti cancer agents. Their article discusses an unconventional ap proach to modify the triphosphate moiety of the nucleotide as opposed to modifying the nucleobase or sugar moiety as observed with conventional chemotherapeutic nucleotides including Fludar abine and AZT. In developing their story, the authors provide new and timely insight into the mechanism of polymerization as well as provide new avenues for drug discovery.

In closing, I am confident that readers will enjoy the contents of the articles provided in this Special Issue as much as I have. While these articles cover much of our knowledge regarding DNA polymerase structure and function, this Special Issue just begins to scratch the surface of nucleic acid chemistry and biology. Over the next several years, we will see an explosion of knowledge in areas including cell based assays that quantify polymerase activity, quantification of dysfunctional polymerase activities in diseased states, and the applica tion of DNA polymerases in biotechnology. Again, the ideas that we view as science fiction today will become the realities of tomorrow!

I would like to express my deep appreciation to all of the authors who have contributed to this very Special Issue. Their extraordinary efforts, keen insight, and overall knowledge of the field has made this issue an outstanding source of knowledge of DNA replication. I was truly fortunate to work with such an illustrious group of scientists. I am also indebted to the scientists that served as reviewers for these articles. Peer review is an important and time consuming commit ment to science, and I am deeply grateful their time, effort, and criticisms as they played a large role in the success of this issue. I wish to thank the members of the BBA editorial board for making this Special Issue possible. I thank all the members of the BBA staff, especially Charity Houston, for their support and help in organizing this issue. Finally, I thank the audience of the BBA for supporting this Special Issue. 